\title{
mHealth Mobile Phone based Patient Compliance System
}

\author{
Divakar Harekal \\ Dept. of Computer Science and \\ Engineering \\ M.S.Ramaiah Institute Of \\ Technology, \\ Bangalore 560054, Karnataka, \\ India
}

\author{
Vijaykumar B.P \\ Dept of Telecomunication \\ Engineering \\ M.S.Ramaiah Institute Of \\ Technology, \\ Bangalore 560054, Karnataka, \\ India
}

\author{
R.Chandrasekhar \\ Department of MCA \\ PES Institute Of Technology \\ Bangalore 560085, Karnataka \\ India
}

\begin{abstract}
This is the era of Mobile communications being deployed for many applications. One such is in the area of health science. This paper presents a unique application developed, called mHealth, that would help the patients, the doctors, the nurses and also the friends of the patients. It is unique in the sense that it alerts patients periodically for intake of medicines by the patients as advocated by the doctors and if the patient fails to do so, a real-time alert will be sent to the doctor. The sequence of operations taken is also entered into a database ported on a mHealth Server for future records. Which patient should take which medicine and at what periodicity can be entered by the doctor into the mHealth Sever.
\end{abstract}

\section{Keywords}

mobile medication, medical compliance, mobile computing, mobile app, patient compliance.

\section{INTRODUCTION}

Small and portable computing devices, such as mobile phones, PDAs, Smart phones, etc., that have recently been pushed into the market, have allowed for social web browsing, cooperation and communication patterns, opened unimaginable opportunities to the future, that were not foreseeable some time ago. Applications have begun to be developed for these computing devices in order to allow for sensor interfaces, data synchronization, Internet browsing, and cooperation. The most targeted domains have been Mobile Commerce, personal assistance and E-shopping. However it is becoming clear that the use of mobile technologies will become quite pervasive in our daily lives and that we need to support development of applications in different critical areas. Some of the critical day to day life activities include family and in house, individual health care and workplace activities. Most dominant among the services includes medical area services. Among health care, individual healthcare with independent management, leads to lots of human error. Outpatient medication administration [2] has been identified as the most error prone procedure amidst the entire medication process. Most of these errors were made when patients bought different prescribed and over-the-counter medicines from several drug stores and use them at home without little or no guidance. Recently, telemonitoring techniques, have been investigated as a cost-effective approach to control quality of care $(\mathrm{QoC})$ for out-patient medication administration. With proper alerts, reminders, dosage quantity, purchase, schedule, etc to the patient and then recording patient's responses, Health Maintenance Organizations (HMO) hope to reduce cost of service, while improving quality of care. In this paper, we introduce mHealth, a smart phone application designed to help patients to avoid medicine administration errors. Our system provides the following facilities by considering all the stake holders, like primarily the patient, pharmacist, medical advisers, dependents, caretakers, etc. The system provides for following functions. (1)Issue medicine in-take reminders (2) Order for Medicines (3) Maintain in-take records. (4) Friends Social Network (5) Compliance meter.

Proposed smart phone application named mHealth is designed to help patients avoid medicine administration errors. This application can run on individual mobiles with some of these critical events, procedures, and reminders to maintain compliance

The term 'Compliance' describes the degree to which the patient correctly follows medical advice.[6] It refers to medication or drug compliance, but it can also apply to other situations such as medical device use, self-care, self-directed exercises, or therapy sessions.[5] The main reasons for noncompliance are:

1. Irregular medicine in-takes due to the patient's busy or erratic lifestyles.

2. Complicated in-take schedules due to many medicines and doses taken by the patient

3. Adverse drug reactions caused by prescriptions obtained from different sources.

4. Lack of knowledge about the proper use of medicines.

5. Lack of consultation with healthcare providers when confusion arises and

6. Lack of monitoring mechanisms to keep track of patient's medicine in-take.

Here both the patient and the health-care provider affect the compliance. Also a coordinated physician-patient relationship is the most important factor in improving compliance, although the high cost of consultation, diagnosis and prescription of medication also plays a major role.

Sending timely reminders, dosage quantity, dietary availability, doctors schedule etc, to the patients and producing proper medicines from the dispenser significantly improve the quality of healthcare from the patient's perspective. From a medical organization's point of view, this approach reduces the cost of manual service, while it also ensures a good quality of care. Hence it is a win-win situation for both the health organizations and the individuals

Equipping such mHealth system, with user interfaces, will help the patient easy access, to recognize the proper medicines and obtain the correct instructions about taking these medicines 


\section{RELATED WORK}

Recently, there has been increasing interest in using mobile phones and wearable sensors for remote health monitoring. On the other hand, using mobile technology to improve patient compliance is still an emerging research area.

For example, Kim and Jeong have used a self-reporting system for the patients to input their blood glucose level, diet and exercise diary using mobile phones. Based on this information, the doctors send their interventions using Short Message Service (SMS)[1]. Logan et al. have proposed a mobile phone based patient monitoring system for managing hypertension in diabetics. The patients are requested to report hypertension measurements twice a day and will receive compliance reminders on their phones if measurements are not received on schedule [2].

All these methods have primarily focus on self-reporting, and minimal non-compliance detection. Other issues that hinder the adoption of self-regulation have been less addressed, and the extent to which these methods can positively influence the patients' compliance is unclear. They are discussed in the scope of our study and understanding

The novelty of mHealth lies in the fact that there is a minimal focus on self-reporting.[4] The application keeps reminding the patient of any particular activity that he is supposed to carry out. It relies mainly on the trust reposed in the patient to follow the regimen set by the doctor.

\section{SYSTEM ARCHITECTURE}

\subsection{System overview}

System depicted in Fig 1 below includes mobile phone with mHealth Server controlled by administrator and the doctor, who prescribes procedure to the patient. mHealth on mobile phone, connect to the server using existing GSM, CDMA with wireless connection with Server and thus gets in-take reminders. User's medicine in-take records. and doctor's prescription and appointment, patient list all will be accessed from the server They are saved in the Server which uses My SQL for database to access data and respond to queries

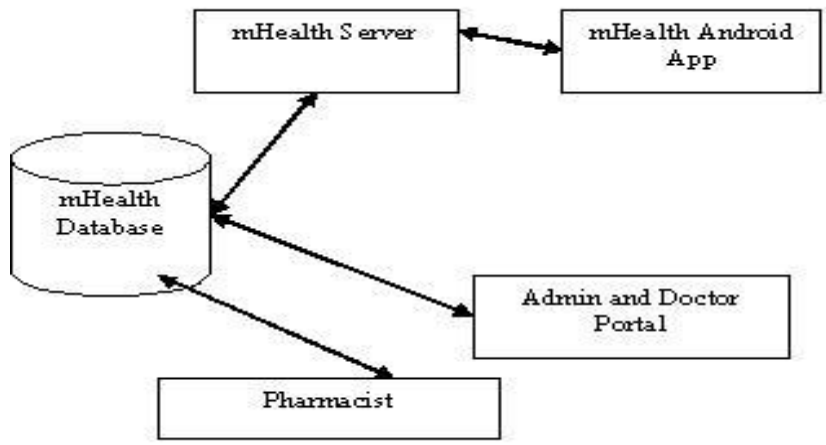

Fig. 1 System Architecture

\subsection{Operational overview}

A scheduler at the Server maintains a event register for compliance, with a monitoring system with dynamic updating capabilities. Such capabilities help to deliver timely alerts, reminders, messages that dynamically alter dosage quantities, request and get doctor's appointments etc can be altered to individual requirements of all stake holders, and enhancements for the future QoC. The response of patient, pharmacist, and doctor is written on to the log file, for appropriate decision making by the individuals. The doctor can login to the portal and check his patient's compliances and keep track of the reminders sent to the patient. The pharmacist can verify the stock of individual patient medicines as per the doctor's prescriptions. The response of the patient is written on to the log file in the server side.

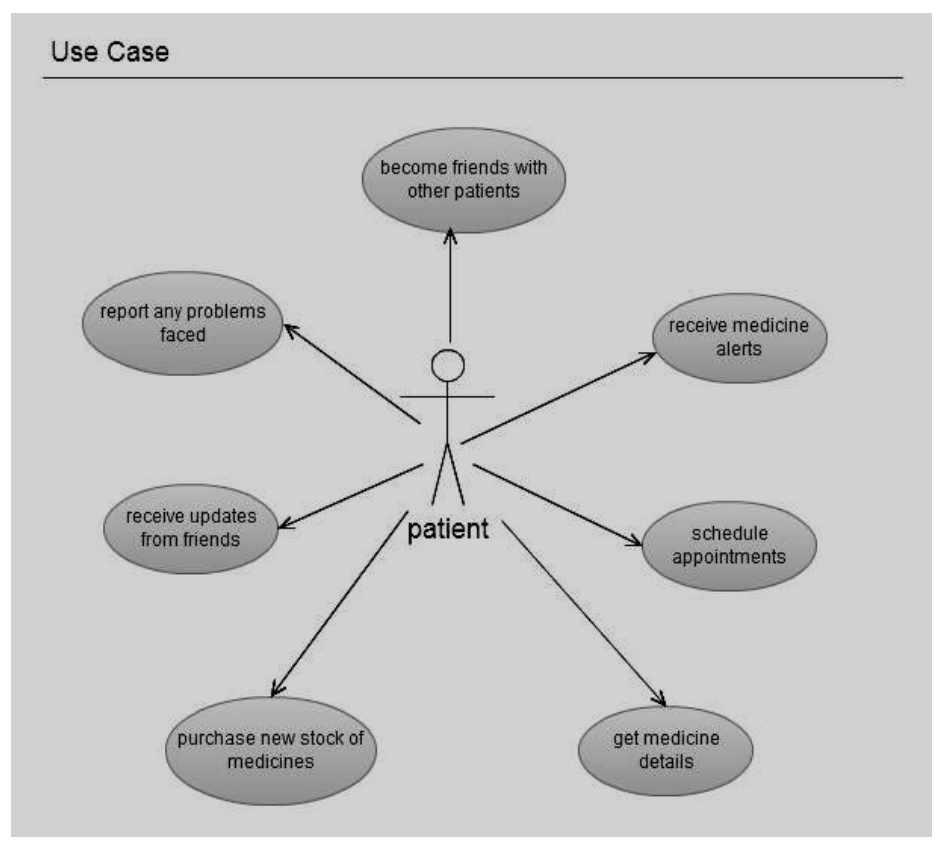

Fig. 2 Patient's Use Case

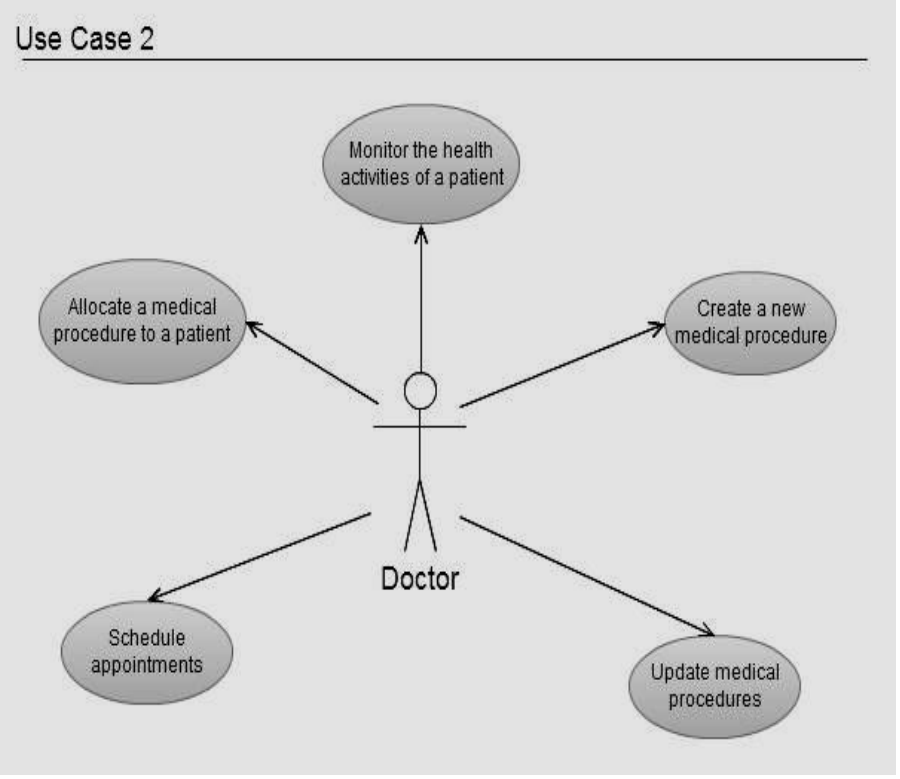

Fig.3 Doctor's Use Case 


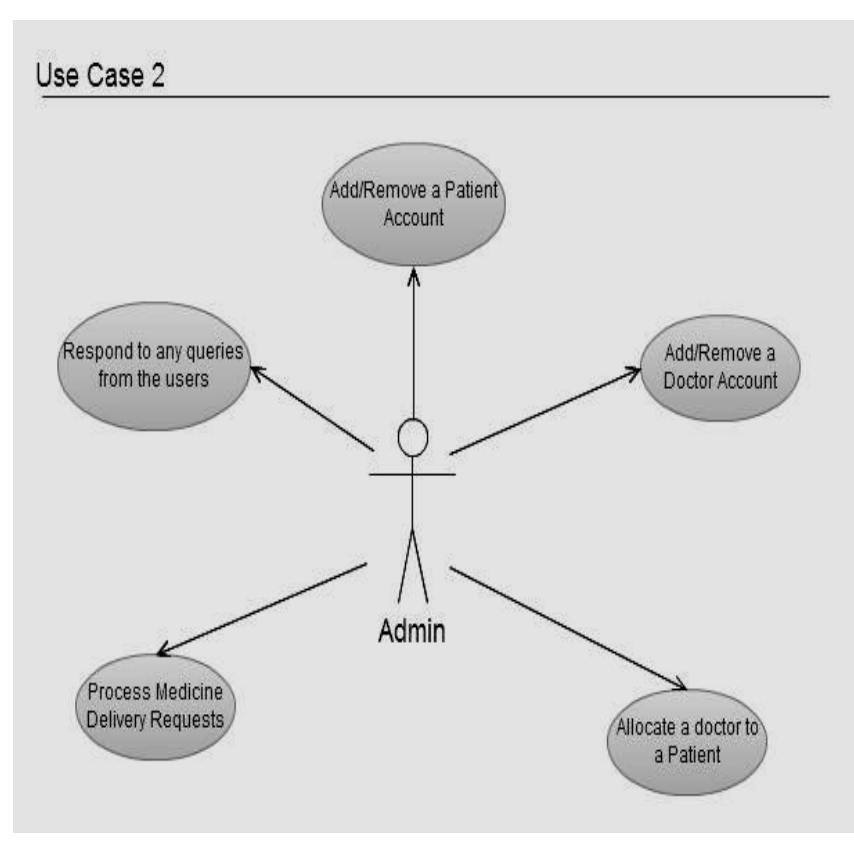

Fig.4 Admin's Use Case

\section{WORKING MODEL OF MHEALTH}

The project mHealth is aimed at being a useful tool in the process of outpatient medication. The doctors can set medical regimens, which have to be adhered to by their patients. The patients receive notifications as per their prescribed regimens, prompting them to perform the necessary activities as prescribed by their doctors. Fig 2, Fig 3, and Fig 4 are use case diagrams that give the functionality required by each of the stakeholders of the system.

\subsection{In-take reminders and response}

The patient would get alerts according to the schedule of compliance prescribed by the doctor. The patient takes the medicine and responds for the same, using a key press or touch screen All activities would be stored in a log file in the server. Suppose if he skips the same medicine for more than two times, then a window is popped up asking the reason for non compliance and the same would be stored in the server for doctors reference and also sends an alert to the doctor

\subsection{Scheduling appointment}

The patient can request for appointment by using mHealth. Rather than going to the hospital for an appointment, it is much easier to do it using mHealth. The request would be updated on to the doctors computer and accordingly an appointment is fixed.

\subsection{Compliance Meter}

In order, to give visual picture of the compliance of the patient, there is a compliance meter. Compliance meter shows the degree of patient compliance with his regime. It is calculated by considering the responses to the reminders and alerts. The patient can look at his compliance levels for each day. A pie chart is displayed containing green color for the percentage of successful compliance and red color for the non-compliance

\subsection{Social Network}

Social network in mHealth includes three major portion:

The more interesting feature is the social network of the patients in the HMO. A patient can find friends who are registered in the particular $\mathrm{HMO}$ and send friend requests to them.

If a patient is complying with schedule prescribed by his doctor, then all his close friends, concerned relatives and QoC friends would get the notification saying your friend is complying with schedule. Even if the patient fixes the appointment or orders a new set of medicines, alerts are sent to his friends. The main intension of this is to motivate other people to follow their regime. A Patient can always use friend updates option to get to know their activities, and also about the same type of diseases.A patient can have a look at his friends list and even modify, if required, with respect to privacy or other personal issues

\subsection{Purchase Medicine}

Suppose the patient is running out stock of specified medicine and needs more stock of medicines, then he can give the order giving the name and quantity of the same with the interactive dialogue with our system. The request would be analyzed by doctor and sent to the pharmacy to replenish the stock and deliver the requested medicine

\subsection{Medicine Details}

It is necessary for a patient to build trust and confidence over the medicine he is taking. mHealth helps to educate the patient by providing the details like effects and quantity in which it is to be taken, standards, costs, dietary, contents etc.

\section{IMPLEMENTATION}

mHealth application is implemented by building various modules with the help of Eclipse on Android 2.3 using a simulator and then tested on Samsung and HTC smart phones. The admin and doctor module was implemented on windows XP using Net Beans. The database has been implemented using My SQL. mHealth application is installed on patient phone The entire operation is shown in Fig 5

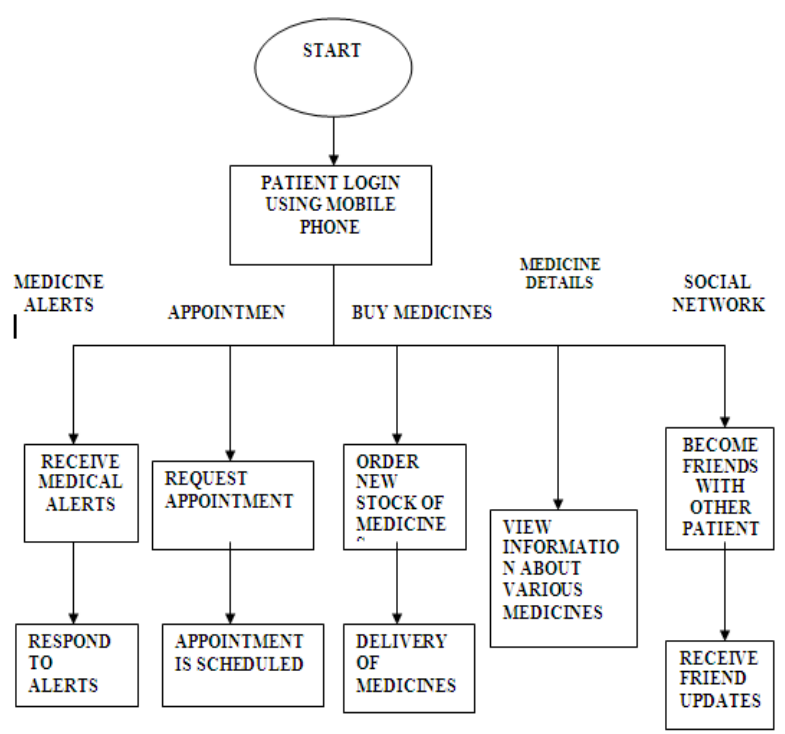

Fig5 Flowchart of mHealth Application

\subsection{Login Module}

The Login module accepts from the user, inputs like username and password and checks them with the stored user account data. If the input is correct, then it provides them access to mHealth. Also subscription option may be provided using 
proper dialogue between user profile and system requirement. Flowchart of the login is given in Fig 6

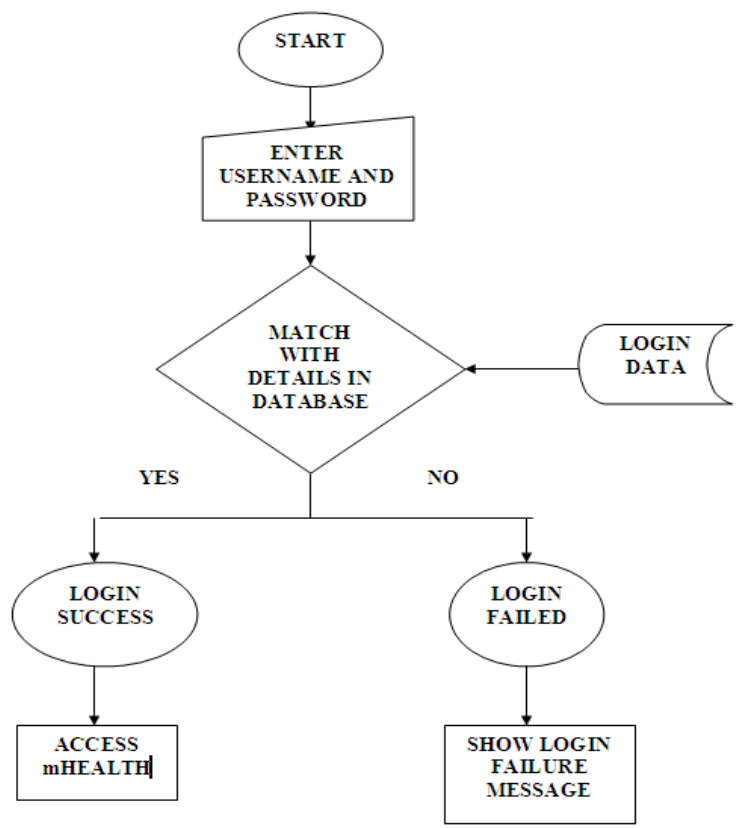

Fig 6 Login Module flowchart

\subsection{Admin Module}

Admin module is hosted on a web server with a front end. It can create or remove user accounts for patients or doctors. mHealth allocates patients to a doctor depending on nature of patient's illness. Also deliver medicines to patients as per their request. A flow chart depicting the admin functions is shown in Fig 7 and login snapshot of admin is shown by Fig 12

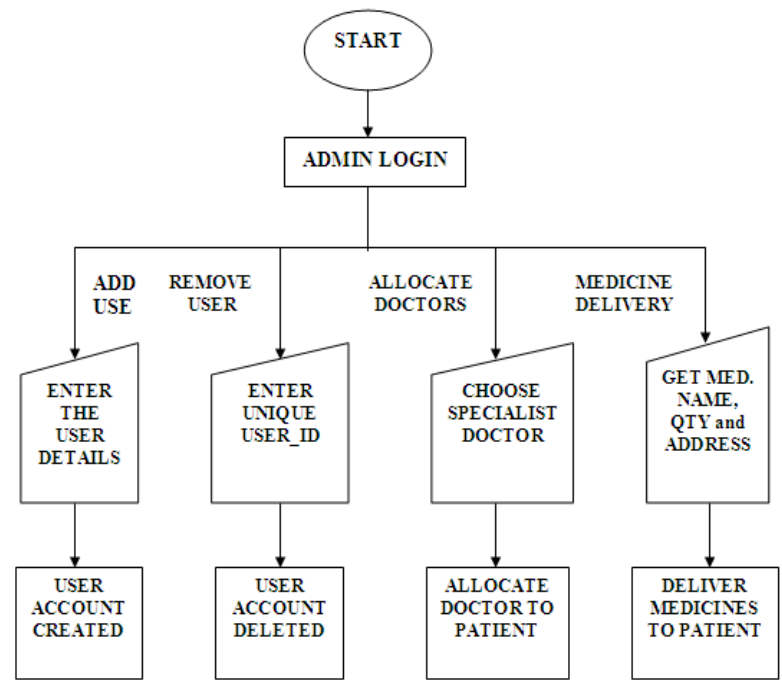

Fig7 Flow chart of Admin Module

\subsection{Doctor Module}

This Module is installed on doctor's system connected to web server. It helps to create medical procedures, which have to be adhered to by the patients. Doctor can monitor the compliance of patient and respond to appointment request sent by the patient Functions available to the doctor is shown in flowchart as in Fig 8

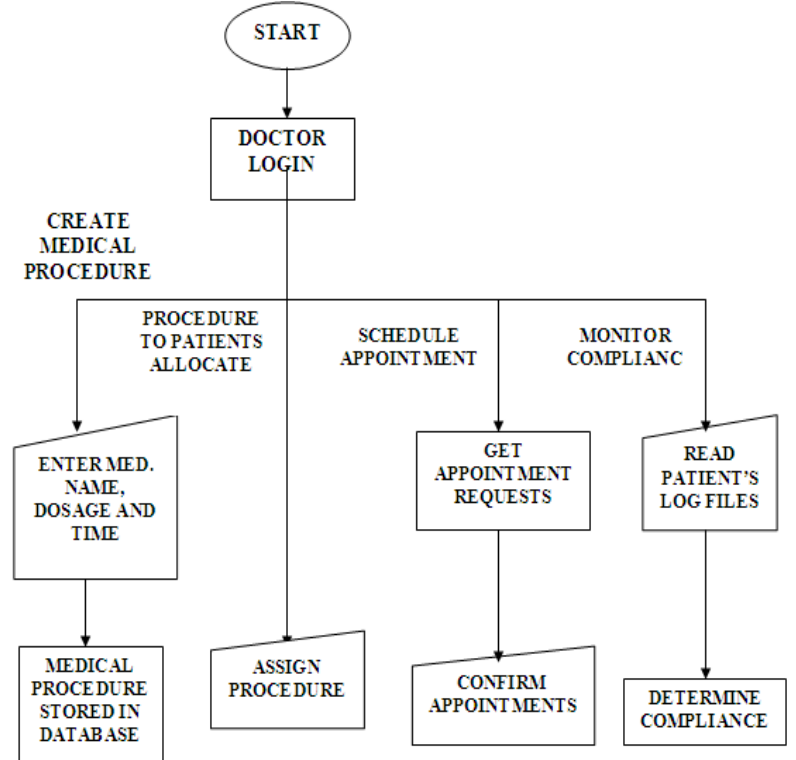

Fig 8 Doctor Module flowchart

\subsection{Appointment Module}

The patient through mHealth, can make a request for appointment with his doctor as depicted by snapshot in Fig 9 . The doctor can respond to request depending on his schedule. After the allotment, date and time is sent as a notification to the patient.

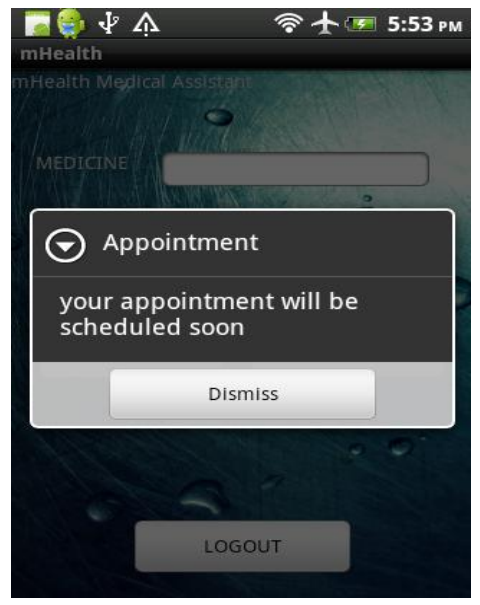

Fig.9 Appointment snapshot

\subsection{Medicine Delivery}

The patient can order only those medicines which are prescribed to him by the doctor. The organization would be responsible for delivery of medicines to the patient. Also a patient can get the bill on his mobile. A delivery response snapshot is shown in Fig 10. 


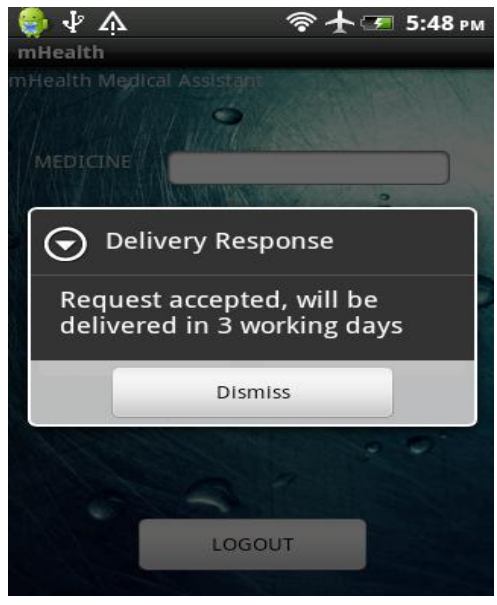

Fig10. Medicine delivery snapshot

\subsection{Friends Network}

Yet another novelty of mHealth is the social network. Each patient can send friend requests. Also each patient can get updates of all his friends activities, their QoC, etc

\subsection{Medical Alerts}

A patient can get all the medicine reminders as suggested by the doctor. The patient can acknowledge the alerts as shown in Fig 11. A patient compliance report is generated. If patient is not complying then he can mention the reason for noncompliance and the same would be updated in the log file of the patient

\subsection{Medicine details}

his module helps to educate the patient. A patient can get to know details of the medicine prescribed to him. Details include the dosage and also the effects of the medicine. Snapshot is shown by Fig 11

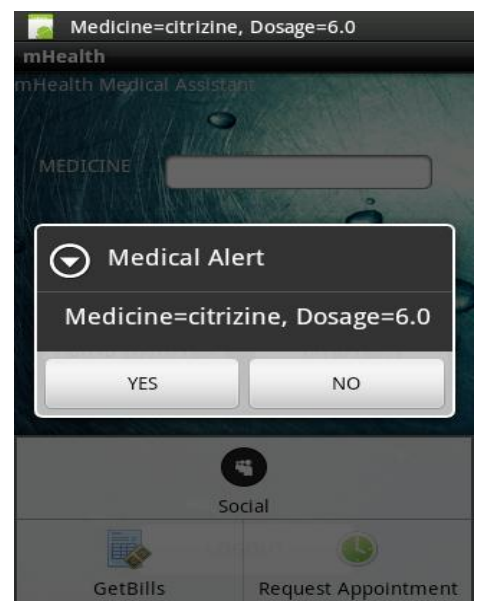

Fig.11. Medical Alert snapshot

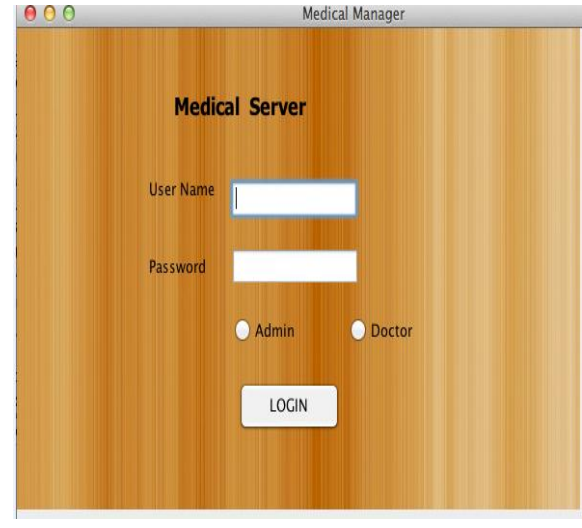

Fig12. Login page for admin and doctor

\subsection{Compliance Meter}

This module gives the graphical representation of the compliance level as shown in Fig 13. Each patient has his compliance level. Needle pointing towards red indicates low compliance and towards green indicates a higher compliance. The main intension is to motivate the user to take medicines regularly.

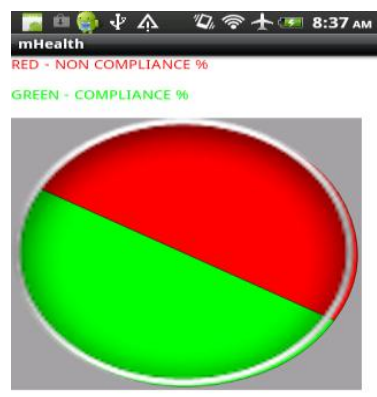

Fig 13 Compliance meter snapshot

\section{EXPERIMENTAL RESULTS}

The mhealth conceived and developed was tested with the cooperation of different stakeholders The medical compliance with the help of the application has been plotted as \%errors in compliance v/s no medical events per day as shown in Fig 14. It is seen that the error rate is significantly reduced with the application. The efficiency of the doctors in monitoring patients has been enhanced by the app, resulting in a higher no of patients that could be effectively monitored this nas been articulated in Fig 15. The Mobile-based Patient Compliance System (MPCS) has also improved the comfort level of the patients and their compliance to over $50 \%$, when the events for compliance is more than 5 /day. Medication efficiency has also marked improvement due to greater no of inputs from the patients to the doctor as shown in Fig 16 


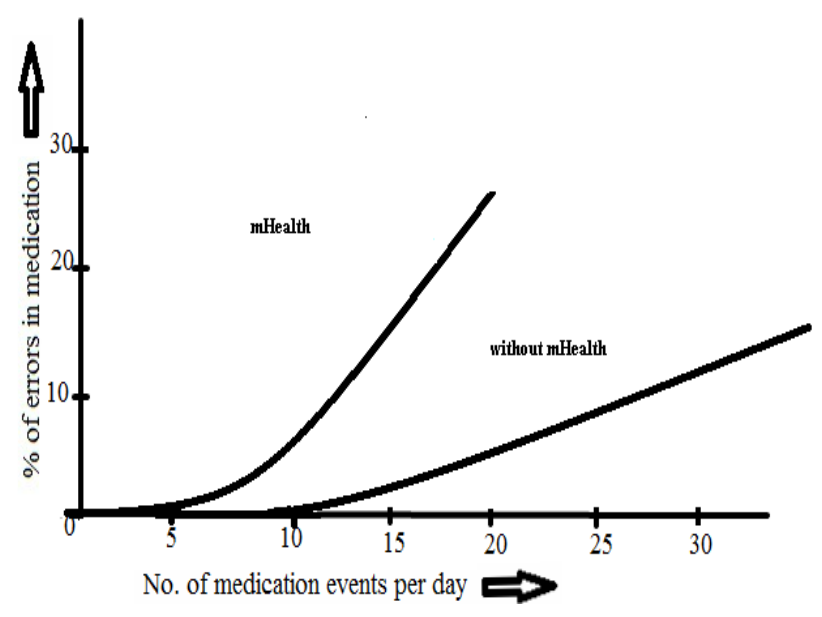

Fig 14

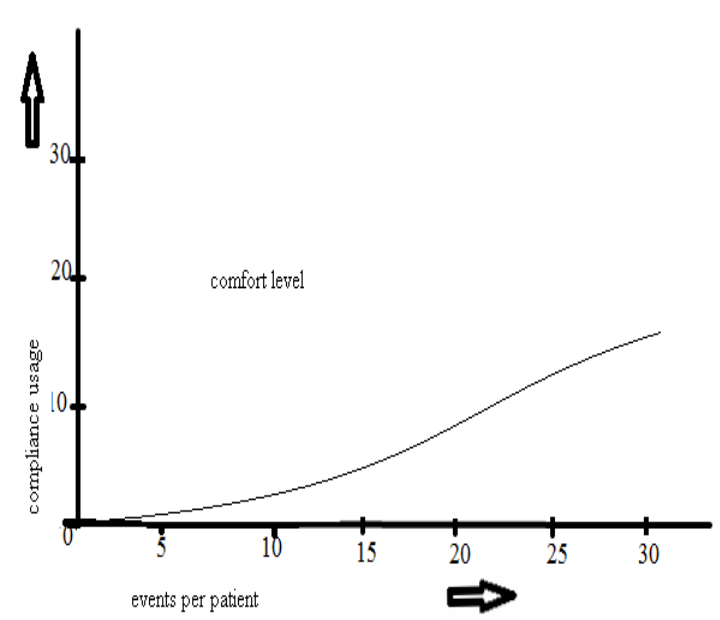

Fig 15

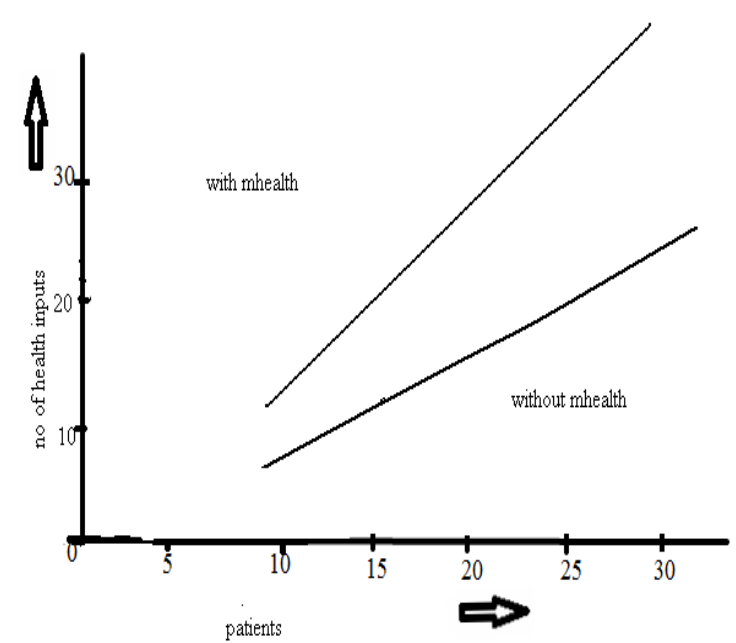

Fig 16

\section{CONCLUSION}

Mobile-based Patient Compliance System (MPCS) is useful for better quality of healthcare (QoC). Our focus is to address the limitations of the self-regulation approach to facilitate its adoption and improve its effectiveness. The novel contribution of mHealth is that we use well-grounded socialbehavioral theories as the design principles for a system built with pervasive mobile technology (primarily mobile phones), to positively influence patient behaviors by reducing compliance obstacles and improving compliance motivations. Earlier work has been based on periodic patient interviews, whereas we plan to use mobile phones for frequent (perhaps even continuous) monitoring of patient health and compliance, with real-time reporting to the provider and rapid feedback to the patient. The system however suffers from deficiency as it still relies on the patient for taking the pill physically and reporting to our system The system can be robust by having some sensor on the capsule to study the patient's activity and gesture, with cognitive intelligence which is a part of future study and research work.

\section{ACKNOWLEGEMENT}

We would like to express our gratitude to Dr. S. Y. Kulkarni, Principal and Professor, M.S. Ramaiah Institute of Technology, and Dr. K.G. Srinivasa, Professor and Head of Department of Computer Science and Engineering, for providing an environment to work

\section{REFERENCES}

[1] H.-S. Kim and H.-S. Jeong. A nurse short message service by cellular phone in type- 2 diabetic patients for six months. Journal of Clinical Nursing, 16(6):1082-1087, May 2007.

[2] A. G. Logan, W. J. McIsaac, A. Tisler, M. J. Irvine, A. Saunders, A. Dunai, C. A. Rizo, D. S. Feig, M. Hamill, M. Trudel, and J. A. Cafazzo. Mobile phone based remote patient monitoring system for management of hypertension in diabetic patients. American Journal of Hypertension, 20(9), 2007.

[3] Guanling Chen, Bo Yan, Minho Shin, David Kotz, Ethan Berke. Mobile Phone Based Patient Compliance System for Chronic Illness Care

[4] [33] J. C. McElnay, C. R. McCallion, F. Al-Deagi, and M. Scott. Self-reported medication non-compliance in the elderly. European Journal of Clinical Pharmacology, 53(3-4):171-178, Dec. 1997.

[5] Adherence to long-term therapies: Evidence for action. World Health Organization Report, 2003.

[6] Amosa B.M.G., Longe O. L and Akinbode O. P. MobilePhone Based Patient Compliance System for Chronic Illness care in JCS\&T Vol. 12 No. 1 